\title{
3. Aussprache und Schlussworte
}

\section{Großvorhaben als Herausforderung für den demokratischen Rechtsstaat}

Biaggini: Liebe Kolleginnen, liebe Kollegen, ich freue mich, dass Sie sich hier wieder eingefunden haben für die Diskussion. Diese ist grob gegliedert in drei Teile. Zunächst geht es um allgemeine Fragen, dann um Fragen der Beschleunigung und der Öffentlichkeitsbeteiligung und zum Schluss um Probleme des Kaskadenmodells.

Engel: Es ist mir eine echte Freude zu sagen: Dies waren zwei vorzügliche Referate. Und auf vorzügliche Referate lässt man sich gern in der Sache ein. Das möchte ich tun mit dem Versuch, in etwas anderen Worten zu sagen, wo meines Erachtens der Kern des normativen Problems liegt. Unsere normativen Vorstellungen, sowohl von Rechtsstaatlichkeit wie von Demokratie, sind von einer Vorstellungswelt geprägt, in der der Gesetzgeber im politischen Prozess zu normativen Entscheidungen kommt und diese normativen Entscheidungen in generell-abstrakte Regeln gießt. Dann haben Individuen nicht die Pflicht, sondern nur die Möglichkeit, von diesem vom Gesetzgeber geschaffenen Raum Gebrauch zu machen. Sie entscheiden sich also zum Beispiel, ein Haus zu bauen, und die Aufgabe der Administration und im nächsten Schritt der Gerichte ist es, diese privatautonome Entscheidung des Individuums auf Übereinstimmung mit den vom Gesetzgeber gesetzten Maßstäben zu überprüfen. In dieser Vorstellungswelt sind sowohl Rechtsstaatlichkeit wie Demokratie völlig problemlos aufgehoben.

Mir scheint, dass sich die Art von Problemen oder von Vorhaben, über die wir heute gesprochen haben, kategorial für dieses Modell nicht eignet. Das ist im Grunde, woran sich beide Referate auf unterschiedliche Weise gerieben haben. Warum ist das so? Weil die Vorhaben, um die es geht, erst in Auseinandersetzung mit einem Vertreter des öffentlichen Interesses so weit konkretisiert werden können, dass man die Frage überhaupt sinnvoll stellen kann: was sind normative Erwartungen an dieses Vorhaben? Derjenige, der etwas will als Privater, kann auch mit dem besten Willen nicht hingehen und in Ruhe das Gesetz studieren, das für einen Möglichkeitsraum nehmen, sich in diesem Möglichkeitsraum bewegen, und dann sehen, ob ihm das gelungen ist oder nicht. Er ist darauf angewiesen, in einem 
frühen Stadium in einen Aushandlungsprozess einzutreten, in dem die öffentlichen und seine privaten Interessen in einen Ausgleich gebracht werden.

Das ist prinzipiell gar kein schlechtes Modell. Nur ist unsere Vorstellung von Rechtsstaatlichkeit und Demokratie auf dieses Modell nicht ausgerichtet. Die sich aufdrängende Lösung wäre, dass alle, die positiv wie negativ betroffen sind, in diesen Aushandlungsprozess einbezogen werden. Dann wäre auch das nahe liegende Ergebnis des Aushandlungsprozesses nicht etwa, dass ja oder nein gesagt wird, sondern, dass diejenigen, die etwas hingeben müssen, also zum Beispiel eine Beeinträchtigung ihres Lebensgefühls, dies zu akzeptieren haben für den Rest ihrer Existenz, jedoch dafür in irgend einer Form kompensiert werden. Die Schwierigkeit, mit der wir hier fertig werden müssen, ist, dass nicht nur unsere Vorstellung von Rechtsstaat und Demokratie ganz anders gebaut ist, sondern, dass wir - selbst wenn wir auf solche Aushandlungsprozesse umstellen wollten, was wir implizit an vielen Stellen tatsächlich tun - ganz unterschiedlich leicht in Verhandlungen einzubringende Interessen vor uns haben. Die typischen Interessen sind ganz asymmetrisch zwischen dem Vorhabenträger, der genau weiß, was er will, der sich das genau durchrechnen kann, und denen verteilt, die positiv wie negativ betroffen sind und die typischerweise diffuse Interessen haben. Diffuse Interessen kann man zwar, wenn man möchte, über Verfahrenslösungen wieder zu integrieren versuchen. Das wäre ein denkbarer Weg, über den wir heute nicht so sehr gesprochen haben. Dann müsste man das in Verbände einfügen und sehen, wie die sich an Verhandlungen beteiligen. Aber die näherliegende Lösung ist, dass dies treuhänderisch, durch einen Vertreter, geschieht, dem man vertraut, dass er das auch redlich macht. Und hier scheint mir das Problem zu liegen: Die Bürger trauen der öffentlichen Hand nicht mehr, dass sie ihr Interesse redlich vertritt.

Groß: Beide Referate folgen, wenn ich es recht verstanden habe, der traditionellen Planungsphilosophie. Über öffentliche Infrastrukturvorhaben wird politisch entschieden, über private Großvorhaben wird unternehmerisch entschieden, und das weitere Planungsverfahren dient im Wesentlichen nur der Detailoptimierung und, mehr oder weniger als Neben- oder zweiter Zweck, der Akzeptanzbeschaffung für die einmal getroffene Grundsatzentscheidung. Ich glaube nicht, dass man heute noch so an Infrastrukturplanung - ich will mich jetzt mal auf den öffentlichen Bereich, der auch der größere ist, beschränken - herangehen kann. Es gibt in unserer dicht besiedelten Landschaft einfach zu viele Gegengründe, im Bereich des Lärmschutzes, im Bereich des Naturschutzes, im Bereich des 
Klimaschutzes und dann auch insbesondere - was immer mehr auch in Stuttgart eine Rolle gespielt hat - bei der Prioritätensetzung der Verwendung öffentlicher Mittel.

Jetzt gibt es, glaube ich, grundsätzlich zwei Möglichkeiten: Entweder wird dies sehr früh auf der politischen Ebene abgearbeitet, so habe ich Herrn Waechter verstanden, und dann das Planungsverfahren weiter wie bisher durchgeführt. Oder es gäbe die Alternative zu sagen: Wir können auf der frühen Ebene, wo auch die Betroffenheiten noch gar nicht so klar sind, das Verfahren über das „Ob“ nicht schon abschließen, sondern wir müssen die Entscheidung auch über das „Ob“ in das Einzelzulassungsverfahren oder jedenfalls in die Stufe unmittelbar vor dem Zulassungsverfahren verlagern. Erst dann sind die konkreten Betroffenheiten hinreichend klar erkennbar, auch die Gegengründe so gut zu gewichten, dass ein entscheidungsoffener Prozess erst möglich wird.

Das wäre dann auch der Punkt, und da kommt die Verknüpfung zum Vormittag hin, wo gegebenenfalls auch Volksabstimmungen sinnvollerweise über Einzelprojekte eingreifen könnten. Da widerspreche ich Herrn Waechter. Ich glaube nicht, dass auf der oberste Ebene, bei der Bedarfsplanung, sinnvoll abgestimmt werden könnte. Ein solcher Bedarfsplan enthält Dutzende von Einzelprojekten, über die ein Volk nur mit ja oder nein abstimmen könnte. Was wäre die Konsequenz eines Neins? Es müsste ein mehrjähriger Planungsprozess neu starten, ohne dass man jetzt weiß, ob das Autobahnteilstück XY Grund für die Ablehnung war oder die Bahntrasse Z. Das scheint mir nicht praktikabel zu sein, sondern man muss einen Weg finden, auf dieser Zwischenstufe, zwischen dem konkreten Zulassungsverfahren und der abstrakten Bedarfsfestlegung, die endgültige Entscheidung des Volkes und dann auch eine funktionierende ergebnisoffene Form der Partizipation einzuführen.

Rauschning: Ich wollte eigentlich gar nichts mehr sagen, doch hat mich manches herausgefordert. Zunächst die Frage: Ist das alles unter den Demokratiebegriff zu fassen? Ich sehe die demokratisch legitimierte Entscheidung als wichtig an auch auf der Verwaltungsebene. Ich halte die Verwaltung für demokratisch legitimiert, und ich wehre mich dagegen, grundsätzlich zu sagen, die Verwaltung sei in diesen Verfahren nicht neutral. Das ist praktisch eine Dienstwidrigkeit, was man da voraussetzt. Das kann ich jedenfalls aus meiner Erfahrung nicht bestätigen. Wenn ein Elektrizitätsversorgungsunternehmen und gegebenenfalls öffentliche Einwänder gegeneinander stehen, dann soll die Behörde das nicht neutral beurteilen? Das würde ich als Unterstellung nicht hinnehmen.

Das ist die nächste Frage: Wer ist denn eigentlich hier beteiligt? Es sind dies der Vorhabenträger und die Betroffenen. Und die werden jeweils, wie 
Herr Mann es sagt, in ihrem Verhältnis und für die Entscheidung in einem rechtsstaatlichen Verfahren beschieden. Es stellt sich die Frage: Ist die Beteiligung Nichtbetroffener eigentlich eine Frage der Demokratie? Es mag sein, dass man auf diese Weise Argumente sammelt für Entscheidungen, die der Abwägung bedürfen oder die im Verwaltungsermessen stehen. Aber dass das generell ein Gebot der Demokratie ist, bezweifele ich. Ich meine auch, dass die Berücksichtigung von Bürgerstimmen außerhalb der Betroffenen über die Frage der Verwendung öffentlicher Mittel auch nicht in unser Demokratiesystem gehört. Wir haben gestern über die Budgethoheit gesprochen. Schließlich hat jeweils das Parlament über die Ausgaben zu entscheiden, und die selbsternannten Vertreter des öffentlichen Interesses, die dann zum Widerstand aufrufen, halte ich nicht für demokratisch legitimiert. Das ist die eine Gruppe, die der sozusagen selbsternannten Vertreter des öffentlichen Interesses. Die zweite Gruppe, bei der ich auch gewisse Probleme habe, ist die der Anhänger des Sankt Florians-Prinzips. Diese sagen z. B: Ich bin natürlich dafür, dass die alternativen Energien die anderen Energieformen zurückdrängen, aber es dürfen in meiner Gegend keine Leitungen dafür gezogen werden. Dass man diese aber ernsthaft berücksichtigt und dass man ihnen vor allen Dingen ein Mitwirkungsrecht einräumen will, halte ich jeweils nicht für ein Gebot der Demokratie.

Saurer: Beide Referenten haben im Begriff des Großvorhabens ja nicht nur planfeststellungsbedürftige Vorhaben, sondern auch Vorhaben im Verwirklichungsmodus der Anlagengenehmigung erfasst. Ich möchte nun anschließen an diese Differenzierung. Deren Kern liegt in der Unterscheidung zwischen vorwiegend öffentlichen und privaten Vorhabenträgern und sie hat unterschiedliche Gestaltungsfreiräume für die zuständigen Behörden zur Folge. Meine Frage betrifft den zweiten Verwirklichungsmodus der Anlagengenehmigung und die hier zu ziehenden Konsequenzen aus der Hinwendung des Verfahrensverständnisses zum Demokratieprinzip. Nach der gesetzlichen Ausgestaltung handelt es sich ja gerade bei der Anlagenzulassung nach dem Bundes-Immissionsschutzgesetz um einen gebundenen Anspruch. Nach tradiertem Verständnis ist dies aus Gründen des Grundrechtsschutzes des zumeist privaten Vorhabenträgers auch genau so geboten. Man kann von einer grundrechtlichen Konstitutionalisierung des Zulassungsanspruchs sprechen. Nun lässt sich spätestens seit den 1990er-Jahren beobachten, dass dieses Verständnis des Zulassungsanspruchs als gebundener Anspruch erodiert, wozu zwei Aspekte maßgeblich beigetragen haben. Zum einen die vordringende Charakterisierung von Großvorhaben durch komplexe, mehrpolige Interessenlagen. Zum anderen, das haben ja auch beide Referenten herausgearbeitet, die 
europäische Stärkung des Verfahrensgedankens. Und nun scheint also ein dritter Faktor hinzuzukommen, der demokratische Faktor. Denn ein demokratisch konnotiertes Verfahrensverständnis verlangt eine Mindestoffenheit des Verwaltungsverfahrens, unabhängig davon, ob man es nun schlicht partizipativ anlegen oder gar weitergehend mit direktdemokratischen Elementen aufladen würde. Diese Mindestoffenheit steht dem gebundenen Verständnis strukturell entgegen. Meine Frage an beide Referenten wäre nun, ob Sie diese Einschätzung teilen, und ob gegebenenfalls das Verständnis des Anlagengenehmigungsanspruchs als gebundener Anspruch auf den Prüfstand zu stellen wäre.

Isensee: Ich gehöre der Staatsrechtslehrervereinigung jetzt vier Jahrzehnte an. Vier Jahrzehnte habe ich Übung, Referate zum Thema „Verfahren“ zu hören. Und ich darf vorab sagen: Die heutigen Referate bilden einen intellektuellen Höhepunkt, einen Höhepunkt an dogmatischer Konsistenz und Subtilität. Nie zuvor führten so viele Stufen zu den Höhen der Differenziertheit, nie zuvor so viele Kaskaden zu den Tiefen verfassungsrechtlicher Begründung. Dennoch bekenne ich, ungeachtet der vielen Referate, die ich gehört habe: ich fühle mich eigentlich immer noch als Fremder auf diesem Feld. Die arcana processualia sind mir noch niemals völlig aufgegangen. Mir bleibt nur die Parallelwertung aus der Laiensphäre:

Der Trend durch alle Tagungen hindurch ist ein Überbietungswettbewerb, das Verfahren zu verfeinern, immer neue Finessen zu finden, den Kreis der virtuell Beteiligten zu erweitern, aber auch das Verfahren zu verselbständigen, abzulösen vom materiellen Recht, auf das es sich eigentlich bezieht. Am Anfang stand „Grundrechtsschutz durch Verfahren“, heute heißt es „Verfahrensgarantie anstelle von Grundrechtsschutz“. Wo sind die materiellen Grundrechte der Betreiber und der Anlieger geblieben, wenn es um die Genehmigung von Großanlagen geht? Wo die materiellen Grundrechte der Eigentümer und der sonst Betroffenen beim bevorstehenden Bau der gewaltigen Überlandleitungen? Wo die Grundrechte der Stromgebührenzahler, die wider Willen die Energiewende bezahlen? Wer die weitere Zerstörung der Landschaft durch Windräder und Großmasten bekämpfen will, braucht sich nicht auf grundrechtliche Positionen wie Gesundheit oder Eigentum zu berufen; es genügt, dass er sich als Naturfreund ausgibt. Die konkrete Betroffenheit im Sinne der grundrechtlichen Schutzpflichten hat sich aufgelöst ins abstrakt Allgemeine, für das jedermann als Naturfreund oder als Wutbürger das Wort führen kann. Eine spontane Öffentlichkeit tritt als Widersacherin oder als Konkurrentin zur staatlich repräsentierten Allgemeinheit auf.

In einem Punkt, Herr Waechter, möchte ich Bedenken anmelden: dass Sie die staatliche Exekutive als von vornherein betroffen und befangen, 
also nicht als neutral im Wettstreit der partikularen Interessen sehen. Die Exekutive ist dem Gemeinwohl verpflichtet; sie interpretiert im Rahmen und nach Maßgabe von Verfassung und Gesetz, was das Gemeinwohl erheischt. Wenn Sie hier eine neutrale Einrichtung erfinden, eine Nebenregierung, dann ist das ein Abbau von Demokratie. Eine Institution, die das Verwaltungsverfahren leitet, aber nicht an Weisungen der Regierung gebunden ist, die nicht zum regulären Verwaltungskörper gehört, die auf einer breiteren demokratischen Basis steht als die Regierung, bricht aus dem Gefüge des parlamentarischen Regierungssystems aus und trägt keine Verantwortung vor der Wählerschaft.

In dem Trend zu immer mehr rechtsstaatlicher Weitung und Verfeinerung des Verfahrens steht der demokratische Freiheitstraum, dass am Ende des Prozesses jedermann der Sachentscheidung zustimmen werde. Man will nicht wahrhaben, dass, wo individuelle Freiheit und Vielfalt der Interessen walten, entschieden - also enttäuscht werden muss.

Die Folge der Verfahrenskomplikation ist die zunehmende Verfahrensdauer. Tendenziell dauern Genehmigungsverfahren für Großvorhaben eine Generation und länger. So mancher, der am Ende des Verfahrens als Wutbürger juristisch verspätet agiert, lag am Anfang, als die regulären Anhörungen stattfanden, noch in den Windeln. Die vita brevis ist überhaupt kein Gesichtspunkt für die Gestaltung des Verfahrensrechts. Die Folge: ein neues Grundrecht dazu, den jeweiligen status quo zu verteidigen gegen mögliche Veränderungen, und diese aufzuhalten oder zu verzögern. Ein katechontisches Grundrecht! Die expansiven Verfahrensvorstellungen können ihr demokratisch-rechtsstaatliches Ziel nie erreichen, das Menschenleben aber können sie verfehlen.

Marti: Auch in der Schweiz sind wir natürlich bei Fragen der Realisierung von Großprojekten hin und her gerissen zwischen den Forderungen nach Beschleunigung einerseits und nach Sicherstellung und Förderung der Akzeptanz andererseits. Wie ist der Stand heute? Bei der Beschleunigung hat vor 12 Jahren ein eigentlicher Paradigmenwechsel stattgefunden bei allen Infrastrukturvorhaben, bei denen der Bund umfassende Gesetzgebungskompetenzen besitzt. Das sind die Eisenbahnen und Seilbahnen, die Luftfahrtanlagen, die Nationalstraßen, sprich Autobahnen, die Schifffahrtanlagen, Rohrleitungen und Elektrizitätsleitungen, Militäranlagen und nachträglich dann auch Kernanlagen. Da war die Situation zuvor so: Völlig uneinheitliche Verfahren, Zersplitterung der Kompetenzen für Teilbelange der Plangenehmigung zwischen Bund und den Kantonen. Und das bot natürlich ein sehr großes Verhinderungspotential für die Kantone, namentlich aufgrund ihrer Raumplanungszuständigkeit, wobei das sehr oft direktdemokratisch abgestützt war. So ist es denn erstaunlich, dass es 
1999 gelungen ist durch das Bundeskoordinationsgesetz (ein Sammelgesetz, das 18 Bundesgesetze in diesen Bereichen änderte) ein einheitliches Verfahren in dem Sinne einzuführen, dass vorgesehen ist, jeweils eine Grundlagenplanung in einem behördenverbindlichen Sachplan des Bundes zu erlassen und dann ein Plangenehmigungsverfahren - und das ist das Wesentliche - konzentriert und zentralisiert bei der zuständigen technischen Bundesverwaltungsbehörde durchzuführen. Da sind dann natürlich auch Fragen aufgetaucht, ob das neutrale Behörden seien. Aber es war der politische Wille, sie einzusetzen als Genehmigungsbehörden, und zwar nach dem Motto: Ein Verfahren, ein Entscheid, ein Rechtsmittel. Wir haben heute den Begriff one-shop-Konzept gehört, vielleicht entspricht unser System diesem Begriff sehr gut. Es ist erstaunlich für ein Land, das man landläufig als erzföderalistisch hält, dass es gelungen ist, ein solches Verfahren einzuführen. Wir könnten in der Schweiz heute also eine ganz neue Eisenbahnlinie vom Bodensee an den Genfersee durch x Kantone bauen mit einer einzigen Plangenehmigungsverfügung des Bundesamtes für Verkehr. Oder in einem Luftkurort könnten wir eine neue Seilbahn bauen mit einer Plangenehmigungsverfügung nicht aus der Region, sondern aus Bern (Bundesamt für Verkehr). Diese ganzen Änderungen haben natürlich die Akzeptanzfrage nicht gerade erleichtert, zumal keine neuen Partizipationsinstrumente eingeführt wurden. Es ist zwar richtig, dass mit der neuen Bundesverfassung von 1999 vorgesehen worden ist, dass Planfeststellungsbeschlüsse durch die Bundesgesetzgebung dem fakultativen Referendum unterstellt werden können. Das ist aber meines Wissens bisher, wie von Herrn Waechter erwähnt, nur bei den Rahmenbewilligungen für Kernanlagen geschehen. Es wäre ja auch problematisch, vor allem dort wo wir, wie jetzt schon mehrfach erwähnt, Grundrechtsansprüche der Träger haben. Übrigens gab es die Möglichkeit, Planfeststellungsbeschlüsse durch Bundesgesetzgebung dem fakultativen Referendum zu unterstellen, schon vor der neuen Bundesverfassung. Es wurde auch schon erwähnt, dass die neue Alpentransversale (NEAT) - noch nach altem Recht durch einen Bundesbeschluss, der dem Referendum unterstellt war, bewilligt wurde; und da kam es ja auch zu einer positiv ausgegangenen Volksabstimmung. Im Übrigen haben wir die traditionellen Mittel des Planungsrechts, also die Einwendungen der Bürger im Rahmen der Bürgerbeteiligung bei Planungen, aber - sofern keine besondere Betroffenheit besteht ohne nachfolgenden Rechtsschutzanspruch. Vor einigen Jahren haben wir sodann auch die Mediation im Verwaltungsverfahrensgesetz des Bundes eingeführt. Die ist aber meines Wissens bei Großprojekten - das wäre ja auch schwierig - noch nie zum Einsatz gekommen. Ein altes Ventil in diesen Bereichen stellt eine andere direktdemokratische Institution der Schweiz dar: die Verfassungsinitiative auf Bundesebene. Diese wurde 
auch schon als Verhinderungsinstrument eingesetzt, beispielsweise als es in den 1980-er Jahren darum ging, einen neuen Waffenplatz im Kanton Schwyz zu verhindern. Damals hat man einen Moorschutzartikel in die Verfassung eingefügt. Oder 10 Jahre später den Alpenschutzartikel, der die Verkehrsverlagerung auf die Bahn verlangt und - darunter leiden Sie (als Automobilisten) vielleicht manchmal - einen weiteren Ausbau der Gotthard-Autobahn verunmöglicht. Im Übrigen haben auch wir eigentlich keine neuen Rezepte. Vielfach wird ein Problem einfach ausgesessen. Ein Beispiel für Aussitzen und Mini-Lösungen sehe ich in meinem Herkunftsort - Sie kennen es vielleicht auch. Es ist diese merkwürdige zweispurige Autobahnbrücke in Schaffhausen über den Rhein, mit 60-Kilometer-Geschwindigkeitsbeschränkung und großer „Bussenfalle“ für alle durchreisenden Touristen - ein Kompromiss nach über zwanzigjährigem politischem Kampf. Danke.

Calliess: Wenn wir auf die Herausforderungen schauen, dann ist es wichtig, auf ein aktuelles Beispiel, die Energiewende und den damit einhergehenden Netzausbau, einzugehen. Insoweit gibt es zwei Herausforderungen, einerseits die Beschleunigung des Netzausbaus, andererseits die Akzeptanzsicherung. Im Sachverständigenrat für Umweltfragen haben wir uns intensiv mit diesem Fragenkreis befasst. Bei der Beschleunigung spielte eine große Rolle, dass im Netzausbau bislang einerseits informelle Prüfungen und andererseits Mehrfachprüfungen stattfinden. Deswegen haben wir dafür plädiert, das Raumordnungsverfahren wegfallen zu lassen und in nur noch zwei formellen Verfahrensstufen, einem Bundesfachplan „Übertragungsnetze“ mit einer Bedarfs- und Alternativenprüfung und einem darauf aufbauenden Planfeststellungsverfahren, zu entscheiden. Dies mit der entsprechenden zweistufigen Öffentlichkeitsbeteiligung: beim Bundesfachplan ist diese ohnehin aus der insoweit gebotenen Strategischen Umweltprüfung (SUP) heraus geboten, im Planfeststellungsverfahren ist sie seit jeher etabliert. Im Übrigen, insoweit kann ich mich kurz fassen, haben wir uns rechtsvergleichend von den Erfahrungen der Schweiz inspirieren lassen, wo im Hinblick auf eine vorhabensbegleitende Einbindung aller Akteure vorbildliche Ansätze vorhanden sind, die sich auch in unser Recht integrieren lassen. Ich brauche das jetzt nicht näher ausführen, manches ist soeben angeklungen. In der Tat sollten wir uns daher nicht auf die uns bekannten Verfahren und Instrumente beschränken. Wir sollten vielmehr stärker vergleichend arbeiten und berücksichtigen, wie es andere Länder machen, wie man dort die Herausforderungen im Rahmen großer Infrastrukturvorhaben löst. Wie generieren andere Länder Akzeptanz? Und wie werden dort Großvorhaben beschleunigt? Vielen Dank. 
Wielinger: Erlauben Sie mir zunächst eine allgemeine Bemerkung. Es ist nicht nur mir aufgefallen, dass in den Erörterungen dieser Tagung bei keinem der Themen Österreich vorgekommen ist. Es hätte sich das etwa bei der direkten Demokratie durchaus angeboten. Ich kann Ihnen sagen: Sie verstoßen nicht gegen das Anschlussverbot im österreichischen Staatsvertrag, wenn Sie das tun würden. Nun, zum Thema. Ich habe mein Berufsleben hauptsächlich in der Verwaltung verbracht und war auch wesentlich an der Schaffung der Novelle zum Allgemeinen Verwaltungsverfahrensgesetz betreffend die Großverfahren in den Jahren 97 und 98 beteiligt. Auch Herr Wiederin, der ebenfalls Mitglied der Vereinigung ist, war dabei. Und es hat sich damals gezeigt, dass das Thema Öffentlichkeit ein ganz anderes ist, je nachdem, welche Möglichkeiten der Mobilisierung der Öffentlichkeit die Rechtsordnung offen lässt. Bis zu der erwähnten Verfahrensnovelle musste nämlich auf jede Einwendung, die in einem Verwaltungsverfahren - vom wem immer und mag sie noch so absurd gewesen sein - erhoben worden war, im Bescheid, der das Verfahren erledigt hat, eingegangen werden. Damit war die Möglichkeit geschaffen, die Entscheidung zu verzögern, ja zu blockieren. Das Ergebnis war: Bei jeder besseren Abfallbeseitigungsanlage, bei jedem besseren Straßenprojekt wurden von den Personen, die an der Verhinderung interessiert waren, Einwendungen organisiert. Das wildeste war ein Verfahren in Oberösterreich mit 40.000 Einwendungen. Seit der Verfahrensnovelle, deren Studium ich anheimstelle, gibt es das nicht mehr. Denn nunmehr muss nicht mehr auf jede Einwendung eingegangen werden. Und es gibt die Möglichkeit, und die hat sehr zur Entschärfung beigetragen, der öffentlichen Erörterung eines Projekts. Das hat zwei Wirkungen. Einerseits Befriedung und andererseits auch die Möglichkeit der Zerstörung von Legenden. Denn wir dürfen eines nicht übersehen: Der Widerstand gegen Großprojekte wird oft um des Konfliktes willen betrieben, weil es immer wieder Leute gibt, die einen Konflikt provozieren, weil ihnen ihre Führungsrolle im Konflikt Sozialprestige vermittelt. Wenn es nun die Möglichkeit gibt, rational über ein Thema zu sprechen, kann es auch zu einer Erhöhung des Grades an Rationalität und zur Zerstörung von Legenden führen. Meine Damen und meine Herren, wir dürfen nicht übersehen, dass es gerade bei Großprojekten in sehr vielen Fällen nicht um Interessen, sondern um den Konflikt als solchen geht. Das sollte man auch bei der Diskussion über Lösungsmöglichkeiten beachten und darauf sehen, dass man Regelungen schafft, die es verhindern, dass Konflikte um des Konfliktes Willen provoziert werden können. Danke.

Schoch: Verehrte Kolleginnen und Kollegen, gestatten Sie mir eine Vorbemerkung und Ausführungen zu drei Punkten. Zur Vorbemerkung: Beiden Referenten danke ich für sachliche Referate, die die Thematik angenom- 
men und reflektiert behandelt haben. Sie sind vor allem nicht modischen Erscheinungen des Zeitgeistes hinterher gehechelt, haben zu den Wirkungen von Öffentlichkeitsbeteiligung keine überzogenen Erwartungen geweckt und haben den Aspekt der „Akzeptanz“ von Entscheidungen ungeachtet der großen Bedeutung - nicht zum Selbstzweck erhoben. Das alles hat einen Grund, der genannt werden muss: Wir haben in unserem Verwaltungsverfahrensrecht mittlerweile einen ziemlich hohen Standard erreicht; Genehmigungsverfahren, Planfeststellungsverfahren oder auch Bauleitplanverfahren sehen vielfach, wie die Referenten gezeigt haben, eine Öffentlichkeitsbeteiligung vor, wir müssen das Rad also nicht neu erfinden. Dies darf einmal deutlich gesagt werden. Wo waren denn die selbsternannten „Parkschützer“ in Stuttgart, als die regulären Verfahren abliefen? Es liegen rechtskräftige Gerichtsentscheidungen vor. Haben die „Parkschützer“ seinerzeit alle geschlafen? Zu dem Punkt von Herrn Isensee komme ich gleich. Zu „Stuttgart 21“ sind rechtsstaatlich korrekt Verfahren mit vielfältigen Beteiligungsmöglichkeiten durchgeführt worden. Wieso ist es, etwas pathetisch formuliert, in unserem Gemeinwesen offenbar nicht mehr möglich, dass sich Verantwortliche zu den Verfahren bekennen und deren Ergebnisse verteidigen? In der Öffentlichkeit wird, verstärkt durch die Medien, so getan, als gebe es in Stuttgart jetzt erstmals die Öffentlichkeitsbeteiligung. Und dann wird Heiner Geißler ,eingeflogen“, der aber doch ganz andere Funktionen erfüllen soll, z. B. Befriedung der Lage. Unabhängig davon müssen wir aber darauf hinweisen, was verfahrensmäßig bereits geleistet worden ist.

Drei Punkte in den Referaten sind kritisch anzusprechen. Der erste Punkt betrifft die Dimension des Faktors „Zeit“. Herr Waechter ist kurz darauf eingegangen; er hat gesagt, im Grunde sei in seinem vierstufigen Konzept auf der letzten Stufe bezüglich einer möglichen Verfahrensbeschleunigung „nichts mehr drin“. Das war mir eine etwas vorschnelle Abhandlung der Problematik. Die viel zu lange Verfahrensdauer ist ein ganz wesentliches Problem; folglich muss überlegt werden, wo auch - auf den vorgelagerten Stufen eine Zeitersparnis erreicht werden kann. Dazu eine Beobachtung: Die Ortsumfahrung (Autobahn) Colmar in Frankreich ist zügig geplant und durchgeführt worden, die neue Straße wird längst genutzt; in Deutschland befänden wir uns bei vergleichbaren Vorhaben noch in Anhörungsterminen. Die lange Verfahrensdauer verursacht zudem Frustrationen bei den Betroffenen; die Leute wissen nach einer allzu langen Zeit gar nicht mehr genau, worum es konkret geht. Die Straffung der Verfahren in zeitlicher Hinsicht ist dringend geboten.

Der zweite Punkt betrifft die Öffentlichkeitsbeteiligung auf den der Verwaltungsentscheidung vorgelagerten Verfahrensstufen. Herr Mann hat 
sich dafür stark gemacht; dieser Ansatz verdient uneingeschränkt Zustimmung. Nach wie vor bestehen zur Öffentlichkeitsbeteiligung auf den vorgelagerten Stufen Defizite, es gibt - nebenbei bemerkt - insoweit auch Rechtsschutzdefizite, aber das war nicht das Thema. Wenn dem so ist, leuchtet mir nicht ein, warum Sie, Herr Mann, auf den der Entscheidung vorgelagerten Stufen die Kategorie „Grundrechtsschutz durch Verfahren“ eingeführt haben; denn dann müssen Sie mit „Betroffenen“ arbeiten. Von beiden Referenten haben wir aber gehört, dass z. B. Linienbestimmungen oder Standortentscheidungen noch gar nicht parzellenscharf getroffen vorgehen können. „Grundrechtsschutz durch Verfahren“ passt da nicht; es muss um eine allgemeine Öffentlichkeitsbeteiligung, wie sie etwa im Änderungsentwurf zu Art. 25 Abs. 3 VwVfG vorgesehen ist, gehen. Mit einer Linienbestimmung oder einer Standortfestlegung, und sei es nur im Groben, fallen wichtige Entscheidungen zum „Ob“ und „Wo“ einer Infrastrukturmaßnahme; dagegen erheben sich nicht selten Fundamentalwiderstände. Bezug nehmen darf ich dazu auch auf den Diskussionsbeitrag von Herrn Wielinger.

Der dritte Punkt, die von Amts wegen erfolgende Information der Öffentlichkeit, ist in beiden Referaten etwas stiefmütterlich behandelt worden. Wenn es richtig ist, dass mit der Beteiligung der Öffentlichkeit in einem Verfahren auch einmal Schluss sein muss und dass mit dem Instrument der „Präklusion“ gearbeitet werden darf, ist die vorherige behördliche Kommunikation mit der Öffentlichkeit umso wichtiger. Dieser Komplex ist bislang „unterbelichtet“. In Bezug auf Reaktionen der Öffentlichkeit kennen wir zwei „Aufreger": Großvorhaben und Lebensmittelskandale. Man schaue sich einmal die zum 1. September 2012 in Kraft getretene Änderung des LFGB (§ 40) an, um zu erkennen, wie die Verwaltung vom Gesetzgeber verpflichtet werden kann, mit der Öffentlichkeit zu kommunizieren. Dann aber, Herr Mann, geht es - entgegen Ihrer Skepsis - nicht ohne Verbände; mit wem denn sonst? Der Einzelne ist, wie allein das Stichwort „Wissensproblem“ deutlich macht, in der Regel komplett überfordert. So hat sich zum Bahnprojekt „Oberrhein drittes und viertes Gleis" eine Bürgerinitiative, der ich mich angeschlossen habe, mit Experten gebildet, die Alternativkonzepte entwickelt hat. Die Arbeit hat im Ergebnis sogar gefruchtet, die offiziellen Pläne werden geändert. Für ein derartiges Vorgehen ist sehr viel „know how“ notwendig; hinzu tritt der Informationstransfer, die Information der Öffentlichkeit. Und dann müssen die Interessen - nicht der „Fundamentalopposition“, sondern der „Wohlmeinenden“ - gebündelt werden. Das gelingt nur über Verbände. Deshalb sehe ich es etwas kritisch, wenn Herr Mann die Verbände eher „mit spitzen Fingern“ angefasst hat. Vielen Dank. 
Lege: Konflikte um der Konflikte Willen - Herr Wielinger, Sie haben das aufgeworfen - werden vielleicht häufig gesucht aus einem Gefühl der Machtlosigkeit heraus, weil einfach keine Mittel vorhanden sind, um sich wirkungsvoll zu wehren.

Ich will mit einem Fall beginnen, dann auf die Thesen von Herrn Waechter eingehen und am Ende zum Rechtsschutz auf den Ebenen 1 und 2, genauer: bei der Bedarfsfeststellung im Eisenbahnrecht kommen und fragen, ob man hier durch eine Stärkung des Rechtsstaats zu mehr Demokratisierung kommen kann. Der Fall ist die ICE-Trasse von Nürnberg nach München. Im Zuge der Verkehrsprojekte „Deutsche Einheit“ gab es Überlegungen, wie diese Strecke geführt werden sollte. Es gab eine Ausbaustrecke über Augsburg und eine Neubaustrecke über Ingolstadt, die beide möglich erschienen. Die Neubaustrecke über Ingolstadt - Sie kennen sie alle - war aus damaliger Sicht erheblich teurer, sie brachte keinen großen Zeitvorteil (die beiden Strecken sind im Grunde ein Parallelogramm), und sie erschien verkehrstechnisch auch weniger sinnvoll. Es sprach also nahezu alles gegen diese Strecke. Zur damaligen Zeit, 1990, war im Bundesschienenwegeausbaugesetz als vordringlicher Bedarf der Ausbau der Strecke „Nürnberg-München“ vorgesehen. Irgendwann, etwa 1993, hieß es dann plötzlich im Bundesschienenwegeausbaugesetz neuer Fassung: „Nürnberg-Ingolstadt-München“. Da wurde also eine Alternativenwahl getroffen, und es gibt nirgends in den Materialen oder sonst wo Gründe für diese Alternativenwahl. Man weiß es einfach nicht, man kann Mutmaßungen aufstellen (Ingolstadt ist der Wahlkreis von Herrn Seehofer, aber gut, das ist eine unsachliche Bemerkung). Rechtsschutz gegen diese Alternativenwahl besteht selbstverständlich nicht. Es handelt sich zwar um ein Einzelfallgesetz, aber es greift ja noch nirgends in Grundrechte ein, insbesondere nicht in irgendein konkret zu benennendes Eigentum, also keine Verfassungsbeschwerde. Und es hat auch niemand eine verwaltungsgerichtliche Klagebefugnis. Man wartet dann also so lange, bis der Planfeststellungsbeschluss am ersten Zwangspunkt fertig ist. Dann besteht die Möglichkeit, diese Entscheidung nachzuprüfen. Aber dann heißt es: Nun gut, die Alternativenwahl auf der Vorstufe wird nicht mehr im Einzelnen überprüft, insofern sei die gerichtliche Prüfungskompetenz per Gesetz verkürzt worden. Kurz und gut, der Rechtsschutz kommt dann viel zu spät, zu einem Zeitpunkt, in dem diese Alternativenwahl gar nicht mehr sinnvoll problematisiert werden kann.

Ich komme jetzt zur These von Herrn Waechter. Sie schreiben im dritten Spiegelstrich am Ende: „Alternativlosigkeit von geplanten Vorhaben lässt sich nur durch Konkurrenz bekämpfen, die es auf den politischen Ebenen 1 und 2 gibt.“ Und dann zwei Spiegelstriche weiter: „Entscheidungen auf den Stufen 1 und 2 sind beim Parlament gut aufgehoben, weil 
dort durch die politische Konkurrenz noch am ehesten Alternativen generiert werden." Aber bei dieser Feststellung des Bedarfs auf der Stufe 1 oder 2 ist auf Seiten des Gesetzgebers der Zwang, Alternativen zu entwickeln, offenbar noch gar nicht vorhanden, oder er wird gar nicht gespürt. Irgendeine Interessengruppe legt die Alternative fest und sagt sich: Nun gut, irgendeine Überprüfung wird später im Rahmen des Planfeststellungsverfahrens stattfinden, aber dann wird das Gericht schon nicht den ganzen Aufwand, der in der Zwischenzeit betrieben wurde, in Frage stellen.

Wie kann man nun dazu kommen, auf dieser vorgelagerten Ebene eine höhere Demokratisierung zu erreichen, also zu erreichen, dass dort Alternativen wirklich generiert und erörtert werden? Mir fällt dazu nur ein: Durch schärferen Rechtsschutz, nämlich einen Rechtsschutz der Gerichte, der sich nicht scheut, auch diese vorgelagerten Entscheidungen zu überprüfen. Und zwar auch im Hinblick auf Kriterien, die den Bürgern wichtig sind, etwa die Wirtschaftlichkeit. Und da finde ich es nun sehr problematisch, dass der VGH Mannheim in der Entscheidung zu Stuttgart 21 mehr oder weniger wörtlich gesagt hat: Die Wirtschaftlichkeit des Projekts entzieht sich der gerichtlichen Kontrolle, kurz: Geld spielt für uns bei der Überprüfung keine Rolle. Denn wenn das so ist, wenn hier von den Gerichten ein Freibrief für Verschwendung erteilt wird, dann entsteht in der Tat bei den Bürgern ein Gefühl der Machtlosigkeit, ein Gefühl, sich nicht wehren zu können - und wenn das Ganze wirtschaftlich auch noch so unsinnig sein mag. Vielen Dank.

Püttner: Ja, meine Damen und Herren, zum Schluss vielleicht noch einmal die Wiederholung, dass wir die Referate mit Vergnügen gehört haben. Zur Sache hat ja Herr Isensee das Nötige ausgeführt, das möchte ich nicht wiederholen. Aber ich muss sagen, zu Herrn Mann: Dieses Kaskadenmodell, das Sie vorgestellt haben, hat doch bei mir einen gewissen Schrecken ausgelöst. Ich sehe ein, dass es gewisse Abstufungen wohl von der Sache her gibt, und verstehe auch, dass man dem Rechnung tragen will. Aber wenn das dazu führen könnte, dass wirklich eine Abschichtung erfolgt, dass also bestimmte Probleme, die auf der ersten Stufe erledigt werden, auf der zweiten Stufe nicht mehr zur Debatte stehen, dann ist das ja recht erfreulich. Aber, Sie haben selbst gesagt, dass das im Zweifel nicht gelingen wird, denn wenn die Verhältnisse sich ändern, dann wird auch die Grundfrage wieder neu aufgerufen. Also fällt selbst die Präklusion als der Hauptvorteil solcher Stufung dann wohl nicht gerade weg, aber er ist nicht so prägnant, wie er sein sollte. Bleiben also nur die Nachteile dieser Stufung. Nachteile nenne ich, dass das Verfahren mehrfach in die Öffentlichkeit gehen muss, dass mehrfach der Aufwand der Information der 
Öffentlichkeit betrieben werden muss, dass es mehrfach den Rechtsschutz gibt, der alles aufhalten kann, dass also eine enorme Verfahrenskomplizierung stattfindet. Ich antworte daher mit der Gegenforderung: Auch bei Großvorhaben sollte es nach Möglichkeit nur eine einheitliche Entscheidung mit einer einmaligen Öffentlichkeitsbeteiligung geben. Damit muss dann auch Schluss sein, damit zügig voran gegangen wird, wie das, soweit ich gehört habe, in anderen Ländern auch der Fall ist. Ich gebe zu, dass dies nicht immer mit geltendem Recht so ganz zu vereinbaren ist. Wir haben auch im Baugesetzbuch mehrfach diese Abstufung, das ist mir bekannt. Aber man sollte doch vielleicht darauf hinarbeiten, dass es möglichst nur einheitliche Entscheidungen gibt. Danke schön.

Biaggini: Bevor wir zu den Schlussworten kommen, möchte ich Ihnen noch ein kleines Geheimnis verraten. Wir haben stillschweigend die Redezeit von drei auf vier Minuten verlängert. Trotz längerer Redezeit ist das „Ampelbild“ etwa dasselbe geblieben. Die Selbstregulierung scheint zu funktionieren. Nun hat bei den Schlussworten Herr Mann zunächst das Wort, dann Herr Waechter.

Mann: Liebe Kolleginnen und Kollegen, lassen Sie mich mein Schlusswort mit einem unserem Tagungsort Kiel geschuldeten maritimen Bild beginnen. In der Diskussion ist mein kleines Segelboot zum Entern freigegeben worden. Einige Enterhaken haben verfangen, dafür sind aber auch Kanonenkugeln, ich denke an die Kanonenkugel von Herrn Lege, an meinem Schiff vorbeigeschossen und haben dafür den Viermaster von Herrn Waechter getroffen. Nachdem der Pulverdampf jetzt verraucht ist, bin ich froh, dass die Masten stehen geblieben sind und einige von Ihnen sogar zur Stabilisierung des Bugs beigetragen haben, damit das Schiffchen noch schnittiger werden kann. Nicht nur für diese, sondern für alle Diskussionsbeiträge sage ich Ihnen Dank. Ich möchte mein Schlusswort nach der Reihenfolge der Beiträge und nicht thematisch gliedern.

Herr Engel, Sie haben auf die Gesetzestreue des Betreibers hingewiesen und auf den Konflikt zwischen seinen frühzeitigen konkreten Planungen und den spät vorgebrachten diffusen Bedenken der Einwender. Daraus folgte ihre Forderung, es bedürfe einer Instanz, der die Bürger vertrauen. Das muss meiner Meinung nach die Verwaltung sein. Bei ihr kommen die Informationen zusammen, aufgrund derer entschieden wird. Ob das nun durch eine Sonderbehörde passiert, wie in der Schweiz oder in Frankreich ich meine, dass Herr Groß einmal über eine solche Sonderbehörde in Frankreich berichtet hat -, oder durch Landesbehörden, ist eine Folgefrage. Für Deutschland scheint mir dies ein föderales Problem zu sein. Im Energiebereich ist es die Bundesnetzagentur für die länderübergreifenden 
Leitungen geworden, aber dass man die Zuständigkeit generell auf eine Behörde konzentriert, wäre mir eher suspekt. Herr Rauschning, ich bin ja vollkommen bei Ihrem Ansatz. Ich habe nicht behauptet, die Verwaltung sei nicht neutral - das kann man von mir als einem deutschen Beamten auch nicht erwarten. Ich habe nur gesagt, es entstehe leicht ein solcher Eindruck für die außen stehenden Einwender, wenn die Verwaltung mit dem Vorhabenträger schon Vorabsprachen im Vorfeld führt, wie es gesetzlich vorgegeben ist. Die demokratische Legitimation habe ich auch nicht verwässert. Was ich versucht habe, ist, die durchaus vertretenen deliberativen oder diskursiven Demokratiemodelle in einer moderaten Form in die bisherigen monistischen Modelle zu integrieren. Ich bin diesen alternativen Demokratiemodellen also nicht gefolgt, sondern ich habe es zu machen versucht wie Johannes Rau: „Versöhnen statt Spalten“.

$\mathrm{Zu}$ Herrn Groß: Ihr Plädoyer dafür, die Entscheidung über das „Ob“ erst dann zu treffen, wenn das Projekt hinreichend konkretisiert ist wenn ich Sie richtig verstanden habe -, läuft ja ein bisschen auf den aktuellen Gesetzesvorschlag hinaus. Ich meine nur, dass da zwei Dinge verbunden werden, die gar nicht zusammengehören. Denn ich kann doch über die Frage des „Ob“ - etwa, ob ich neue Energieleitungen brauche, die von der Nordsee bis hinunter nach Bayern gehen - besser unabhängig von einem konkreten Projekt diskutieren. Ich sehe es daher gerade umgekehrt. Wenn ein Vorhaben schon hinreichend konkretisiert ist, dann diskutieren die Leute die Frage des „Ob“ doch gar nicht mehr offen, weil sie nämlich wissen, dass das Endlager in ihre Kommune kommen soll. In diesem Stadium kann man nicht mehr unbefangen über ein „Ob“ diskutieren. Man muss also das „ $\mathrm{Ob}^{\text {“ }} \mathrm{zu}$ einem Zeitpunkt diskutieren, zu dem das Projekt gerade noch nicht konkretisiert ist. Daher habe ich die abstrakte Stufe der Bedarfserörterung vorgeschlagen. Ich gebe zu, dass meine Sichtweise auf diese Dinge vielleicht etwas negativ ist. Viele von Ihnen haben möglicherweise eine idealistischere Vorstellung von der Öffentlichkeitsbeteiligung und dem Erörterungstermin. Mein Bild, und das mag auch meine Argumentation sicher beeinflussen, ist davon geprägt, dass ich seit nunmehr 10 Jahren im 7. Senat des Niedersächsischen OVG sitze und wir dort für Straßenplanung, Wasserstraßenplanung, Bergrecht, Abfallverbrennungsanlagen, Energierecht und Atomrecht zuständig sind. „Atomrecht“, das heißt in Niedersachsen: Castor-Transporte, Schacht Konrad, Endlager Gorleben, Asse II und Zwischenlager Unterweser. In meiner Praxiserfahrung habe ich also ständig Großvorhaben auf dem Tisch, bei denen es verhärtete Fronten gibt. Verfahren auf dem Vergleichswege zu erledigen, wie das ja sonst auch in der Verwaltungsgerichtsbarkeit möglich ist, scheidet hier aus. Ich erinnere mich nur an einen Fall in den letzten 10 Jahren, wo das gelungen ist. Das ging es um eine Müllverbrennungsanlage, bei der 
die Kläger damit zufrieden waren, dass sie vorher angerufen werden und die Wäsche reinholen können, wenn es einen Störfall gibt. Den Regelfall aber bilden Gutachterschlachten, Demonstranten vor dem Gericht, aufgebrachte Zuhörer im Sitzungssaal und Befangenheitsanträge gegen die Richterbank. Das ist mein Erfahrungshorizont, unter dem ich dieses Referat geschrieben habe und daraus speist sich, das gebe ich gerne $\mathrm{zu}$, meine Einschätzung von den Erfolgsaussichten einer moderierenden Öffentlichkeitsbeteiligung in einem Erörterungstermin. Deswegen möchte ich den Erörterungstermin auf die in ihren Rechten Betroffenen reduziert wissen. Zudem: Bei allem Zutrauen in die Problemlösungskraft der von Herrn Waechter angesprochenen Mediation möchte ich doch bezweifeln, ob es angesichts der Radikalisierung und Verhärtung der Fronten bei der Genehmigung von Großprojekten einem Mediator gelingen kann, den schmalen Bereich des Konsenses zu treffen. Es wird immer Gruppen geben, für die alles andere als die Null-Lösung, also Projektverhinderung, nicht konsensfähig ist. Und denen können Sie - es tut mir leid, wenn das jetzt so nach 19. Jahrhundert klingt - nur mit einer staatlichen Verwaltungsentscheidung begegnen, die notfalls auch vollstreckbar ist. Natürlich muss man überlegen, wie wir möglichst viele mit ins Boot nehmen können. Aber „Akzeptanz“ wird zu Recht in den Sozialwissenschaften diskutiert, das ist keine rechtsdogmatische Kategorie. Ich habe sie dem rechtsstaatlichen Gedanken des fairen Verfahrens zugeordnet. Herr Saurer, Ihre Frage ging, wenn ich Sie richtig verstanden habe, dahin, ob auch bei der industriellen Anlagenzulassung so ein Kaskadenmodell entstehen soll. Wir haben das im Grunde heutzutage schon, indem - wenn ich das jetzt richtig überblicke - solche großen Vorhaben in ein Raumordnungsverfahren gegeben werden, um ihre Raumverträglichkeit zu prüfen. De lege ferenda könnte man auch solche Verfahren mit in dieses Konzept hineinnehmen.

$\mathrm{Zu}$ Herrn Isensee: Es bestätigt mich, wenn Sie auf die Grundrechte der Betreiber und der Nutzer aufmerksam machen, denn das habe ich in meinem Referat auch schon getan. Es ging mir ja gerade darum, dass nicht immer nur auf die Wutbürger gesehen, sondern erkannt wird, dass auch der Vorhabenträger Grundrechte hat, es sei denn, es ist ein öffentlicher Vorhabenträger. Ich denke, wir sind auch nahe beieinander, wenn ich die Reichweite der Beteiligung im Erörterungstermin auf die Rechtsbetroffenen reduzieren möchte. Der Kreis der Teilnahmeberechtigten bestimmt sich nach der Reichweite der subjektiv-öffentlichen Rechte. Ist kein subjektiv-öffentliches Recht ersichtlich, das betroffen sein könnte, lässt sich auch aus den verfassungsrechtlichen Rechtsschutzgarantien kein Anspruch auf Beteiligung ableiten, weil diese als formale Sicherung der Freiheit eben eine Betroffenheit dieser Freiheit voraussetzen. Sehr zutreffend 
finde ich die Beschreibung von Günter Gaentzsch in der Festschrift Sellner. Er war der Leiter des Erörterungstermins am Frankfurter Flughafen und hat geschätzt, dass wohl 90 Prozent des Gesprochenen bei dem Erörterungstermin für die Entscheidung - ich habe es mir extra aufgeschrieben - „rechtlich völlig unerheblich“ war. Und das entspricht auch meiner Erfahrung. Nur ein Beispiel: Es wäre sinnvoll, von einem Erörterungstermin ein Ergebnisprotokoll zu machen. Die Einwender wollen aber immer ein Wortlautprotokoll. Welchen Vorteil bringt denn ein Wortlautprotokoll? Nun, man kann damit nach Hause gehen und sagen: Seht her, ich habe mich dagegen gewehrt. Also nur Schaulaufen für die eigene Klientel. Diese Beobachtung spricht im Grunde auch Ihren Einwand an, dass es vielen Leuten allein um das Dagegensein geht, nicht aber um ein konstruktives Sachergebnis. Demgegenüber wollen diejenigen, deren Grundstücke betroffen sind, vernünftig argumentieren, und ich bin überzeugt, dass auch die Verwaltung und die Betreiber sehr daran interessiert sind, zu vernünftigen Lösungen zu kommen, die dann auch für die Betroffenen akzeptabel sind. Zu Ihrem zweiten Einwand, Herr Isensee: Zeitgerechtigkeit! Ist das nicht ein grundsätzliches Problem politischer Entscheidungen? Diskutieren wir das nicht auch auf anderen Feldern, nur unter dem Stichwort der „Generationengerechtigkeit“? Als Ende der 50er-Jahre die Entscheidung für Atomkraft gefallen ist, da durfte ich auch nicht mitbestimmen.

$\mathrm{Zu}$ Herrn Calliess und seinen Stichwörtern „NABEG, EnWG, Beschleunigung". Ja, das NABEG ist ein guter Versuch, Beschleunigung und Öffentlichkeitsbeteiligung zu verbinden. Mir ist da aber schon zu viel Öffentlichkeitsbeteiligung eingebaut. Ich habe das im Referat ausgeführt. Auch die Idee mit der Bundesfachplanung, die das Raumordnungsverfahren ersetzt, ist ebenfalls gut. Aber das ist doch nur für länderübergreifende und grenzüberschreitende Leitungen vorgesehen. Dafür ist es auch sinnvoll, wenn eine Bundesbehörde die Planung für das Bundesgebiet macht. Genauso wie es wünschenswert wäre, wenn wir eine bundesweite Flughafenplanung hätten. Tendenziell bin ich aber eher kritisch, dem Bund nun sämtliche Planungsaufgaben zu übertragen. Herr Schoch, Sie haben hervorgehoben, dass es in der aktuellen Diskussion auch darum geht, Errungenschaften zu verteidigen. Da stimme ich Ihnen zu. Der von Ihnen eingeforderte Grundrechtsschutz durch Verfahren findet in meinem Modell bei der Planfeststellung, beim Erörterungstermin statt. Nicht aber bei der Bedarfsprüfung, weil ich da keine Rechtsbetroffenheit sehe. Ambivalent ist es, wenn man Grobtrassierungen festlegt. Denn wenn bei der Grobtrassierung beispielsweise klar wird, ob die Linie durch das Sauerland geht oder an der Rheinschiene vorbei, dann kann ich als Bürger bereits erkennen, ob ich möglicherweise betroffen bin oder nicht. Ich 
würde das an dieser Stelle aber immer noch nicht ausreichen lassen, um dann Klagerechte zu geben. Stichwort Verbandsbeteiligung: Ich habe die Verbände nicht mit spitzen Fingern angefasst, wie Sie meinen, ich habe sie gar nicht angefasst. Verbände haben eine wichtige Bündelungsfunktion. Sie sollten meines Erachtens auf allen drei Stufen beteiligt sein. Nach der Trianel-Entscheidung des EuGH werden die Verbände bei uns in Zukunft eine noch größere Bedeutung haben, weil sie dann wahrscheinlich überall auch Verbandsklagerechte bekommen werden.

Abschließend zu Herrn Püttner: Das Kaskadenmodell, haben Sie gesagt, verlängert ja alles, und das führt zu einer Mehrfachöffentlichkeitsbeteiligung. Herr Püttner, insofern muss ich Sie auf den Boden der Tatsachen zurückholen. Eine solche Mehrfachbeteiligung haben wir doch jetzt schon. Seit der SUP-Richtlinie müssen wir bei allen Planungen die Öffentlichkeit beteiligen. Eine nur einmalige Bürgerbeteiligung, wie sie Ihnen vorschwebt, ist eigentlich gar nicht mehr möglich, wenn man Entscheidungen planerisch abschichtet. Mir ging es ja gerade darum, Auswüchse wie die sechs oder sieben Stufen der Beteiligung, die wir jetzt bei den Energieleitungen haben, auf drei luzide Stufen zu reduzieren, bei denen klar ist, wann es um Bedarf, die Grobtrassierung oder die Planfeststellung geht. In dem Kaskadenmodell kann man abschichten, wenn man Präklusion einführt. Das zementiert aber nicht: Wenn ich etwa bei der Bedarfsprüfung feststelle, dass für ein Vorhaben ein Bedarf besteht, dann kann man bei der Planrechtfertigung hinterher nicht mehr sagen: „Wir brauchen das Projekt nicht“. Das ist festgelegt. Erst recht bei einer gesetzlichen Bedarfsfeststellung. Die Behörde kann aber in der Abwägung feststellen, dass zwar ein Bedarf besteht, aber genau an dieser konkreten Stelle etwa Umweltbelange so gewichtig sind, dass er in der Abwägung überwunden wird. Das aber macht in meinem Modell die Behörde. Ich öffne nicht wieder das Tor und lasse zu, dass der Erörterungstermin mit erneuten grundsätzlichen Auseinandersetzungen über das „Ob“ überfrachtet wird. Mir erscheint die Reduktion des Erörterungstermins auf die Rechtsbetroffenen auch deshalb sinnvoll, weil einige Kollegen momen$\tan$ in vielen Rechtsbereichen resignierend die Arme heben und vor den Zuständen kapitulieren, indem sie resümieren, das Erörterungsverfahren bringe ja sowieso nichts, also sei es abzuschaffen. Damit verletzt man aber den Grundrechtsschutz durch Verfahren derjenigen, deren Grundstücke von dem Vorhaben konkret betroffen sind.

Liebe Kolleginnen und Kollegen, ich meine, unsere Aufgabe als Staatsrechtslehrer in dieser Diskussion sollte es sein, Orientierungslinien vorzugeben, die für Konsistenz und Klarheit bei der Bewältigung der einzelnen Sachfragen im Kontext von Großvorhaben sorgen. Ob die Frist für die Auslegung der Unterlagen nun einen Monat oder zwei Monate beträgt, 
oder ob im Internet oder in der Wochenendbeilage der Tageszeitung darauf aufmerksam gemacht werden soll - damit soll sich der Deutsche Juristentag beschäftigen. Ich wollte mit meinem Segelschiffchen vielmehr ein Modellboot in die Staatsrechtslehrerwerft geben, an dem wir alle zusammen arbeiten können. Einige von Ihnen haben ja bereits heute damit begonnen. Wenn am Ende dann ein schnittiger Katamaran zum Stapellauf kommt, bin ich sehr zufrieden. Vielen Dank!

Waechter: Ich hoffe, unser Segelboot ist nicht die Gorch Fock, die im Augenblick im Tirpitzhafen liegt, denn der geht es ja derzeit nicht besonders gut. (Mann: Wird aber generalüberholt.) Ich möchte beginnen mit der Frage: politisch und nicht politisch, mit anderen Worten mit der Differenz Demokratie und Rechtsstaat. Das liegt ja meinen Ausführungen und, wenn ich es recht verstanden habe, auch denen von Herrn Mann zu Grunde. Und das wirft natürlich zunächst die Frage auf, Herr Engel hat sie angesprochen: Kann man einzelne Entscheidungen überhaupt in dieser Weise qualifizieren? Natürlich habe ich mir darüber Gedanken gemacht, und ich glaube eine mögliche Konkretisierung wäre, dass man sagt, in beiden Fällen, bei sachlichen Entscheidungen und bei, in Anführungsstrichen, „politischen“ Entscheidungen, geht es um Reduktion von Komplexität und um Optimierung. Der Unterschied ist, dass die Fähigkeit zur Reduktion dieser Komplexität bei politischen Entscheidungen darauf beruht, dass man über besondere Kenntnisse und Fähigkeiten in Bezug auf Präferenzen in der Bevölkerung verfügt, und bei sachlichen Entscheidungen verfügt man über besondere Erkenntnisse über Fakten und nicht über Präferenzeinstellungen. Damit, meine ich, kann man diese Ebenen etwas sortieren. Der zweite Punkt ist, dass ich nicht davon ausgegangen bin, dass Öffentlichkeitsbeteiligung vom Demokratieprinzip geboten sei. Das bin ich schon deswegen nicht, weil ich ja auf dem Hintergrund der Diskussion der 80-er Jahre spreche, und in den 80-er Jahren war ein Thema, das heute offenbar nicht mehr virulent ist - verständlicherweise, wie ich finde -, die demokratische Legitimation innerhalb der Privatwirtschaft, insbesondere bei Großvorhaben. Das heißt, die dahinter stehende Frage war: Gehorchen Polis, also Staat, und oikos, also Gesellschaft, denselben Steuerungsprinzipien? Soll das so sein? Ich meine, das Versiegen dieser Diskussion zeigt, dass es sinnvoll ist, dass verschiedene Steuerungsprinzipien Anwendung finden. Das ist zwar im Verlaufe der letzten 2000 Jahre in der Rechtsphilosophie immer wieder unterschiedlich gesehen worden, aber ich glaube, im Augenblick kann man das so festhalten. Und auch deswegen die Scheidung nach eher politischen und eher sachlichen Stufen. Und dem entspricht eben auch die Unterscheidung zwischen wertgeprägtem und nutzengeprägtem Protest. Und schließlich 
kann man dazu noch sagen - das ist in den Beiträgen nicht wesentlich angesprochen worden -, wenn man das Referendum in den Blick nimmt: Es wäre ja seltsam, wenn wir bisher immer davon gesprochen haben, dass wir die Entscheidung über Großvorhaben durch Verfahrensrecht rationalisieren wollen, jetzt plötzlich dieses ganze Verfahren wegzuschlagen mit einer Dezision. Und das Gleiche gilt in Bezug auf die Funktion von Gutachtern. Ganz viele Großvorhabenentscheidungen hängen, nach meinem Eindruck, stark von Gutachteraussagen ab. Es wäre auch für den Vorhabenträger wenig einleuchtend - nachdem er möglicherweise ein ganzes Dutzend von Gutachtern aufgeboten hat, die ihm bescheinigen: Die Sache ist in Ordnung -, das Ergebnis dann einer politischen Entscheidung auszusetzen, die sagt, das interessiert alles nicht. Das vorweg.

Herr Engel, Sie haben gesagt, wir brauchen eine Stelle, der die Bürger trauen können. Das habe ich ja versucht zu erreichen, und das leitet über zu der Frage, die von Herrn Rauschning und auch von Herrn Isensee angesprochen worden ist: Neutralität der Verwaltung. Natürlich habe ich darüber keine soziologischen Erhebungen. Aber, erstens habe ich die Beobachtung, dass zumindest in einem Wohlfahrtsstaat die politische Spitze ein Interesse daran hat, dass wohlfahrtsrelevante Entscheidungen von der Verwaltung getragen werden. Zweitens: Natürlich hat jeder Beamte, der etwas werden will, einen gewissen vorauseilenden Gehorsam gegenüber dem, was er als Meinung des Hauses kennt. Das ist doch ganz zweifellos so. In der Literatur wird darüber hinaus vorgebracht: Wenn die Verwaltung Zielfindungsaufgaben hat - und natürlich muss sie sich überlegen, wenn sie von dem Großvorhabenträger im Vorfeld der ganzen Sache angesprochen wird: Was hältst du davon? -, dann muss sich die politische Spitze darüber klar werden, was sie will. Dazu wird sie häufig auch die nachgeordneten Abteilungen und Referate befragen. Das heißt, es findet ein Zielfindungsprozess statt. Dass hinterher keine wirkliche Offenheit mehr da ist, wenn man einmal diesen Zielfindungsprozess durchlaufen hat, ist doch relativ naheliegend. Und ich meine, Sie alle haben Absolventen, die in den Beruf gehen. Und Sie alle kennen das, wenn Sie zum ersten Mal danach mit jenen sprechen, die in die Verwaltung eingetreten sind. Was wird ihnen gesagt? Du sollst nicht prüfen, $o b$ das geht, du sollst prüfen, wie das geht. Das zeigt doch die Sache. Der Untersuchungsbericht zur Asse ist - für mich als Niedersachsen - ein einziger Sumpf von Nichtneutralität, wenn man es mal ganz brutal sagen will. Herr Isensee hat dann in diesem Zusammenhang auch noch gesagt, dass ich eine Nebenregierung installieren möchte. Ich habe ja bewusst diese Neutralität auf die Verfahrensbehörde beschränkt und nicht auf die Entscheidungsbehörde erstreckt. Das hätte ich auch tun können, davon haben mich aber mehrere Dinge abgehalten. Erstens einmal der gemischte 
Charakter des Raumordnungsverfahrens, also der dritten Stufe, wo doch noch ein bisschen politischer Charakter dabei ist. Ich wahre also da die traditionelle demokratische Legitimation. Und zum anderen eine aristotelisch geprägte Vorsicht: Ich will nicht die absolut beste Verfassung oder Lösung propagieren, sondern die relativ beste, die auch eine politische Anschlussfähigkeit hat und Chancen auf Verwirklichung. Im Übrigen: Nebenregierung ist traditionell ja der Effekt der Gewaltenteilung. Vor Montesquieu hat man so gesprochen, wie Sie das getan haben. Aber spätestens in den Federalist Papers haben doch die Amerikaner gesagt: Es geht gerade darum, Interessen so zu institutionalisieren, dass sie gegeneinander wirken. Das ist die Idee der Nebenregierung. Gewaltenteilung ist immer Nebenregierung. Es kommt darauf an, dies im Detail auszubalancieren. Was die Verfahrensdauer angeht, ich meine, das ist ein Vorgriff auf die Beschleunigungsfrage. Man muss sich auch die Lebensdauer der Vorhaben anschauen. Wenn man das recherchiert, dann beginnt das ungefähr bei 50 Jahren für ein Kohlekraftwerk, wenn die Technik erneuert werden muss, und reicht bis weit über 100 Jahre bei Infrastrukturvorhaben. Dann relativeren sich auch die Verfahrenszeiten, glaube ich. Herr Groß, die Idee, die Frage des „Ob“ vor das Zulassungsverfahren zu ziehen, dazu hat ja Herr Mann schon etwas gesagt. Ich glaube, dass es richtig ist, wenn man es auf die Ebene der Bedarfsfrage zieht, dann wirkt die Bedarfsplanung quasi wie ein Schleier des Nichtwissens. Das ist immerhin ein kleiner Gewinn. Dass der Volksentscheid auf der Bedarfsplanebene im Prinzip nicht sinnvoll ist, finde ich auch. Die Lösung vieler hier angesprochener Fragen liegt in einer Veränderung der politischen Kultur: Bei uns hat bisher die Opposition diese Aufgabe nicht erkannt. Und das ist ein wesentlicher Punkt. Natürlich muss die Opposition Alternativen vorlegen, das ist ihre originäre Aufgabe. Und wenn dann die Opposition eine echte Alternative vorlegt, dann kann vielleicht auch eine Volksabstimmung, der ich aber an sich nicht zuneige, über so einen Bedarfsplan eine sinnvolle inhaltliche Aufgabe haben, dass man nämlich das andere alternative Konzept will. Zu der Neutralitätsfrage von Herrn Rauschning habe ich bereits Stellung genommen. Hinsichtlich der Frage von Herrn Saurer möchte ich einfach auf Herrn Mann verweisen. Ja, Herr Marti, wir sind uns vollkommen einig. Sie haben gesagt, man muss die Beschleunigung erreichen durch Konzentration und Straffung und durch Zentralisierung. Es ist ja auch so, dass viele Verfahrensbehörden sowohl personell wie fachlich überfordert sind. Wenn nicht allzu häufig ein Großverfahren kommt, muss eine Behörde sich umfangreich einarbeiten. Wenn man da eine landeszentrale oder gegebenenfalls, wenn das kompetenzrechtlich möglich ist, eine bundeszentrale Behörde hätte, die dann als Verfahrensbehörde dienstleistend dieses Verfahren abwickelt - und in einer Neutralität, wie ich sie versucht 
habe zu konstruieren -, dann würde viel Zeit gewonnen werden können. Und diese Behörde wäre auch fachkompetent. Ja, das gilt auch für Herrn Calliess. Im Prinzip finde ich diese Systematisierung der Planungsschritte im NABEG nicht schlecht. Das Problem ist, dass wir möglicherweise ein bisschen Schwierigkeiten mit der Kompetenzordnung kriegen. Aber jedenfalls könnte man einige dieser Gedanken auf der Landesebene reproduzieren. Dieser Unsinn von paralleler Linienbestimmung und Raumordnungsverfahren jedenfalls - das kann man ziemlich eindeutig sagen sollte abgeschafft werden. Herr Wielinger, ich habe natürlich auch versucht, nach Österreich zu schauen, aber die Schweiz hat nun einmal die Marke auf unmittelbare Demokratie. Darum kommt man nicht herum. Im Übrigen, die Berücksichtigungspflicht in Deutschland geht ja nicht auf die einzelne Einwendung, sondern wir fassen ja immer ganz viele Einwendungen zusammen und berücksichtigen nur den Belang als solchen. Wenn ich Sie recht verstanden habe, haben Sie pro Öffentlichkeit des Erörterungstermins gesprochen - da bin ich mir aber nicht ganz sicher und haben sich gleichzeitig gegen potentielle Konfliktsucher gewandt. Aber wenn es da wirklich ein erhebliches Potential gäbe, die wären ja froh um die Öffentlichkeit. Denn politische Profilierung braucht Öffentlichkeit. Und ob das dann nicht gerade kontraproduktiv wäre für eine solche von Ihnen als existent unterstellte Gruppe, eine stärkere Öffentlichkeit herzustellen, da bin ich mir sehr unsicher. Herr Schoch, ich mache in Frankreich immer Urlaub und führe dann gerne Gespräche mit Bürgermeistern, wenn ich im chambre d'hôte logiere. Ich habe den Eindruck, die haben eine so unterschiedliche Verwaltungskultur, dass man das schwer übertragen kann. Zur Lebensdauer der Anlagen und der Notwendigkeit von Beschleunigung habe ich schon etwas gesagt. Information von Amtes wegen, da stimme ich Ihnen völlig zu, das wäre schön, wenn die Behörden das Internet mehr nutzen und viel mehr einstellen würden. Diese Geheimnistuerei, das weckt ja gerade das Misstrauen, insbesondere diese Zurückhaltung von Gutachten usw. Das halte ich auch im Bauleitplanungsverfahren, wo ich als Richter überwiegend tätig bin, für groben Unsinn. Dass nicht zwingend alle Gutachten offengelegt werden müssen führt zwangsläufig dazu, dass die Bürger die Verwaltung für befangen halten, auch wenn sie es gar nicht sein sollte. Verbände, das ist natürlich ein bisschen problematisch, weil die Interessen der Betroffenen und die der Verbände auseinandergehen. Da würde ich also Vorsicht walten lassen und erst einmal abwarten, wohin sich die Lage entwickelt. Herr Lege, im Grunde fordern Sie ja eine Begründungspflicht für den Gesetzgeber, jedenfalls wenn er Bedarfspläne macht. Ich glaube nicht, dass das sonderlich sinnvoll ist, weil das ja wieder auf die judizielle Überprüfung dieser Bedarfspläne hinausläuft, die aber aus meiner Sicht in der Tat einen über- 
wiegend politischen Charakter haben. Deswegen würde ich auch insoweit lieber darauf setzen, dass die politische Kultur sich so ändern muss, dass die Opposition ihre Alternativen präsentiert, und dann gerät automatisch die Mehrheit, die ihren Bedarfsplan beschließt, unter Begründungszwang. Und insofern ist das Problem nicht nur auf der Seite der Staatsrechtslehrer, sondern das Problem ist auch auf der Seite der Bürger und der Parteien, dass sie auf diese Großvorhaben in adäquater Weise reagieren und eine entsprechende politische Kultur sich erarbeiten. Und dann kann man nicht solche Bedarfsplanfeststellungen im Bundestag stillschweigend als Opposition an sich vorbeigehen lassen. Dass Geld keine Rolle spielen soll, das ist schön. Aber der Bürger glaubt es nicht, schlicht und einfach. Und selbst wenn Gerichte bereit sind - wir hatten ja zuletzt den Fall Kraftwerk Datteln, da war das Gericht ja bereit, das Geld keine Rolle spielen zu lassen - aber der Bürger glaubt es nicht, und bei Befangenheit kommt es eben auch darauf an, was geglaubt wird. Schließlich Herr Püttner. Sie sprechen für eine umfassende Konzentrationswirkung. Das ist natürlich wünschenswert, das ist völlig klar. Ich habe ja auch beim Rechtsschutz gesagt, ich hätte gerne konzentrierten Rechtsschutz. Aber erstens kann man konzentrieren nur insoweit, wie die Struktur der Entscheidungen zueinander passt. Wenn sie unterschiedlich strukturierte Entscheidungsmuster haben wie zwischen politischen Entscheidungen und rechtsstaatlichen Entscheidungen, also Sachentscheidungen, dann können sie nicht dieselben Maßstäbe zur Kontrolle anlegen. Das erschwert so eine Konzentration. Im Übrigen stimme ich völlig mit Ihnen überein: Der Nachteil - das habe ich ja auch gesagt - jedenfalls bei der Konzentration des Rechtsschutzes ist, dass Implizitprüfungen bisher schwächer ausfallen. Das wäre meines Erachtens aber wiederum eine Frage der Gerichtskultur, dass die Gerichte erkennen müssen, wenn wir einen konzentrierten Rechtsschutz haben, wie auch jetzt nach dem neuen NABEG, wo die Trassenfestlegung nur im Rahmen der Planfeststellung angegriffen werden kann, dann muss man auch die Implizitprüfung ein bisschen gründlicher machen. Ich danke Ihnen für Ihre Aufmerksamkeit und der Vereinigung für die Gelegenheit zum Vortrag. 